\title{
tentative d'interprétation des déformations observées aux tunnels du Fréjus et du Gran Sasso
}

\author{
par \\ P. Berest, P. Habib, Nguyen Minh Duc \\ Laboratoire de Mécanique des Solides \\ (Groupe Commun École Polytechnique, ENSM Paris, ENPC \\ Équipe de Recherche associée au CNRS) \\ École Polytechnique - Palaiseau - France.
}

\begin{abstract}
Quelles sont les caractéristiques mécaniques d'un massif rocheux comme celui où a été creusé le tunnel du Fréjus? La réponse à cette question est tout à fait nécessaire si l'on veut déterminer par le calcul la courbe de convergence du rocher et il est bien évident que plus la définition mécanique du comportement de la roche sera précise, meilleure sera la courbe caractéristique obtenue. Jusqu'à présent, il ne semble pas qu'une telle courbe ait fait l'objet de détermination expérimentale directe, et pourtant elle est essentielle pour le calcul du soutènement d'un tunnel. II est donc extrêmement tentant de profiter du creusement de tunnels comme ceux du Fréjus et du Gran Sasso pour essayer de répondre à une question qui, on le verra au cours de cette étude, laisse l'ingénieur encore assez désarmé.
\end{abstract}

\section{a) Tunnel de Fréjus}

Les calcschistes du Mont Fréjus apparaissent, d'après les essais en laboratoire effectués sur les échantillons prélevés du côté français et du côté italien, comme un matériau assez constant sur une dizaine de kilomètres. Les résultats des essais mécaniques sur des carottes obtenues par sondage indiquent des résistances à la compression simple de l'ordre de $100 \mathrm{MPa}$ et un comportement fragile, ce qui correspond en général à des courbes intrinsèques très ouvertes au voisinage de l'origine. Les modules d'élasticité sont de l'ordre de $50000 \mathrm{MPa}$ et les effets différés sont insignifiants avant la rupture. $\mathrm{Ce}$ matériau est donc très résistant. Cependant il est probable qu'il n'est pas représentatif du massif lui-même, car ce dernier est fortement fissuré; la schistosité très apparente, inclinée au front à $30^{\circ}$ sur l'horizontale modifie de toute évidence les propriétés de l'ensemble, et cela d'autant plus que des traces de graphite diminuent le frottement entre les facettes des fissures de la roche. D'une façon empirique, on peut dire qu'un observateur averti devant un tel massif peut estimer sa résistance à la compression à $30 \mathrm{MPa}$ et son angle de frottement interne à $30^{\circ}$ pour tenir compte à la fois des essais, de la dilatance et de la présence du graphite. On peut estimer le module d'élasticité du massif à $20000 \mathrm{MPa}$, peut-être même moins, lorsque les fissures peuvent s'ouvrir, Mais au sein du massif, sous un recouvrement de plusieurs centaines de mètres, les fissures sont complètement fermées : la vitesse de propagation des ondes sonores (parallèlement à la schistosité) est de l'ordre de $4000 \mathrm{~m} / \mathrm{s}$ à $5000 \mathrm{~m} / \mathrm{s}$ dans le massif sain, alors qu'autour de la galerie dans la zone desserrée elle diminue jusqu'à près de $2000 \mathrm{~m} / \mathrm{s}$. Sans vouloir comparer module statique et module dynamique, il apparaît une réduction de plus de 4 du module d'élasticité dynamique. Pour un calcul, une basse valeur du module d'élasticité n'est évidemment acceptable que pour une détente de la roche à partir d'un fort serrage initial. Par exemple au moment de l'ouverture de la galerie, la contrainte normale à la paroi du tunnel s'annule, on peut donc admettre dans la direction radiale un très bas module d'élasticité; par contre la contrainte tangentielle autour du tunnel augmeñte par rapport à l'état initial. Le module de déformation dans cette direction est donc élevé. La question apparaît ainsi comme bien compliquée. De toutes façons, si l'on estime que l'effet d'échelle peut minorer au tiers la résistance et à la moitié le module d'élasticité, on peut se demander quelle est la signification des essais de laboratoire et penser qu'on n'est pas loin de l'arbitraire.

Les calcschistes du Fréjus sont anisotropes d'une façon évidente. La schistosité n'est pas rigoureusement plane; à l'échelle centimétrique elle est même très tourmentée, tout en donnant une impression générale d'orientation. Cette anisotropie est très nette sur les diagrammes de convergence (fig. 1); la contraction du tunnel dans le sens perpendiculaire à la schistosité est plus grande que celle qui se produit dans le plan de schistosité; selon les sections examinées ces variations vont de $+30 \%$ à $+100 \%$. En définitive, l'anisotropie de déformation paraît peu de chose si on la compare à l'incertitude apportée par 

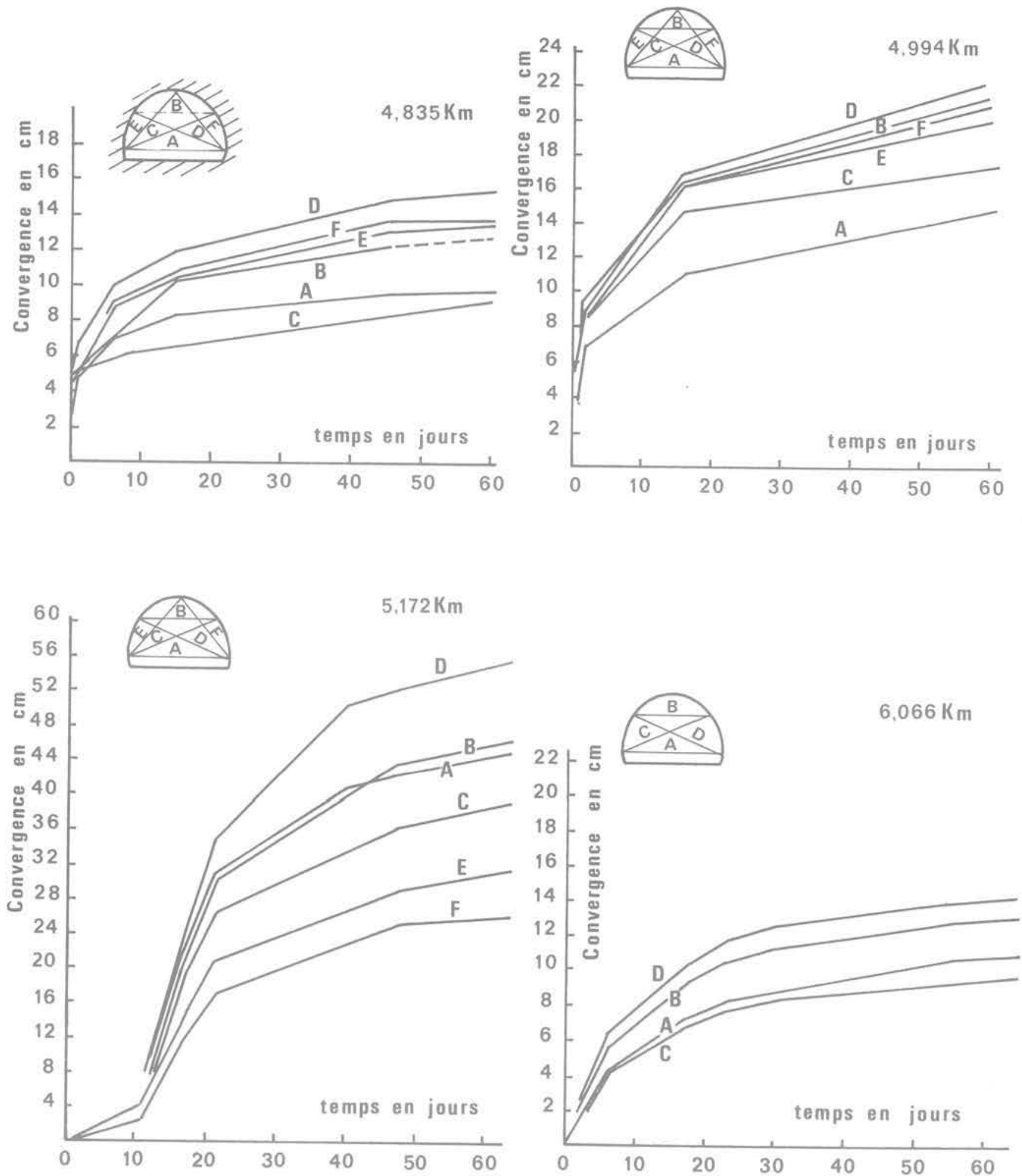

Fig. 1 Anisotropie de la convergence.

l'effet d'échelle, et sans nier son existence, on peut presque négliger cette information en première approximation si on se limite à une évaluation du simple au double pour les déplacements : on verra plus loin que la précision ne va même pas jusque là.

Examinons, maintenant, l'homogénéité du massif. On a dit précédemment que rien de particulièrement net n'avait pu être décelé sur les échantillons prélevés en sondage; cependant l'aspect des parois de la galerie varie d'un point à un autre et les convergences moyennes sont différentes et présentent des effets différés sans qu'une corrélation avec la hauteur du recouvrement (fig. 2) apparaisse clairement. Certes, les plus grandes déformations ont été observées pour des recouvrements supérieurs à $800 \mathrm{~m}$, mais des convergences variant du simple au double ont été mesurées pour des recouvrements constants de $700 \mathrm{~m}$ et le plus grand recouvrement n'a pas été associé aux plus 


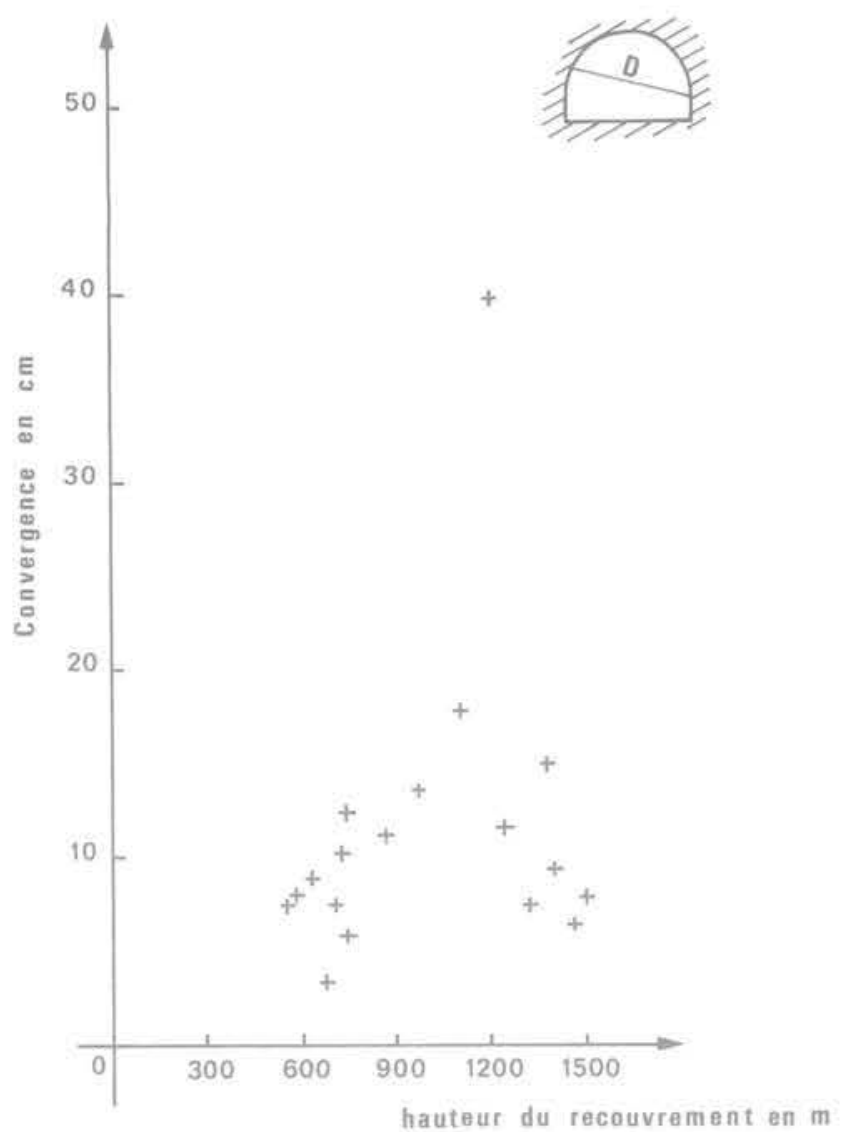

Fig. 2 Convergence dे 1 mois en fonction de la hauteur du recouvrement.

grandes convergences. Deux explications sont possibles. La qualité du massif varie d'un point à un autre; cette explication avait de toute évidence la faveur du chantier, et l'on peut dire même des deux chantiers. Son évidence saute aux yeux: en fonction de l'avancement il a fallu adapter les modes de travail, les soutènements, etc. pour maintenir des parois dont l'état de fragmentation était visiblement différent. On remarquera cependant qu'une variation diamétrale, (c'est-à-dire une convergence), supérieure à 2 ou $3 \mathrm{~cm}$ signale obligatoirement que le massif rocheux est entré dans le domaine plastique. Ainsi avec un recouvrement de $1000 \mathrm{~m}$, équivalent à une contrainte hydrostatique de $25 \mathrm{MPa}$ et un module d'élasticité de $20000 \mathrm{MPa}$, il vient avec $R=11 \mathrm{~m}$ :

$$
\Delta \mathrm{R}=\mathrm{R} \frac{1+\nu}{\mathrm{E}} \mathrm{p} \# 1 \mathrm{~cm}
$$

alors que la contrainte tangentielle à la paroi est de $50 \mathrm{MPa}$, voisine ou supérieure à la résistance. Lorsque la convergence dépasse notablement cette valeur, un certain volume de roche près de la paroi est entré en rupture. Une mesure des contraintes tangentielles, par exemple par un essai au vérin plat, ne permet pas alors de mesurer la contrainte maximale subie par la roche et est tout au plus une mesure de la résistance résiduelle de la roche. De la même façon, on peut dire qu'il est très difficile de décider de la résistance initiale du massif, comme on l'a fait précédemment, rien qu'en le regardant de l'intérieur de la galerie, c'est-à-dire après la perturbation apportée par la perforation. De sorte qu'une autre explication de l'inhomogénéité du massif peut être avancée, qui consiste à dire que la

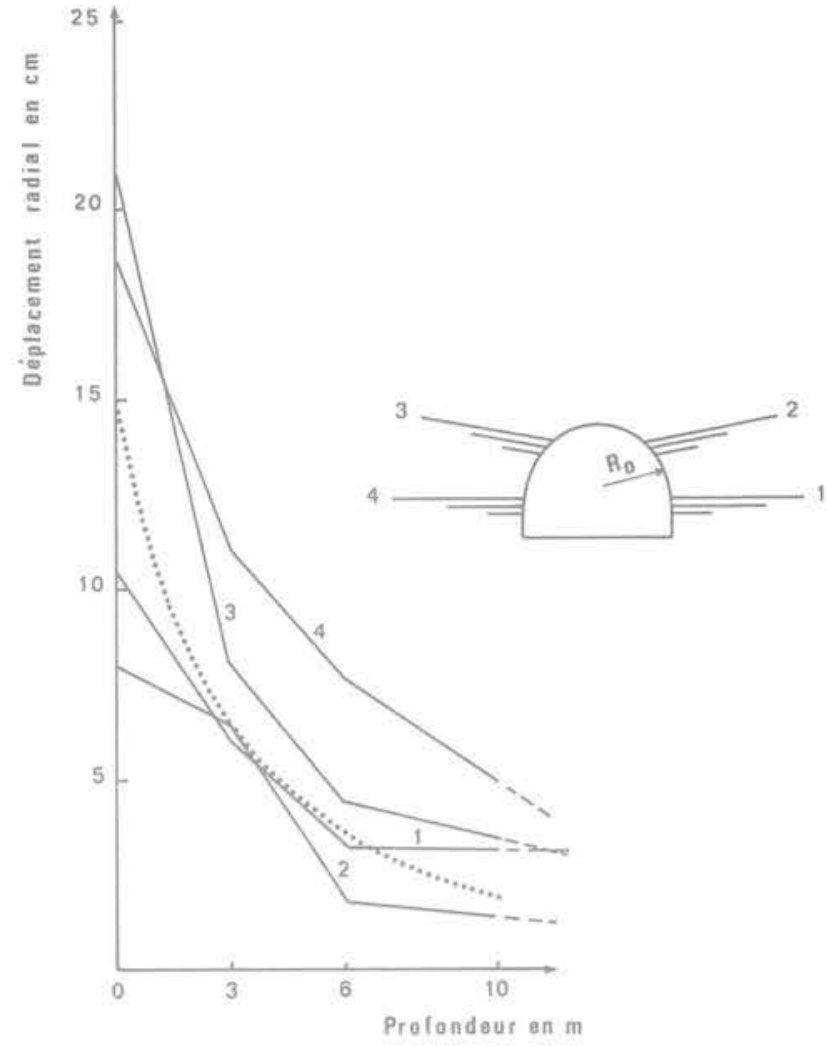

Fig. 3 Déplacement radial en profondeur au tunnel du Fréjus.

qualité de la roche est presque partout la même, mais que les contraintes naturelles ne sont pas homogènes. En perçant le tunnel dans une zone surcontrainte on libère davantage d'énergie et on a des déformations plus grandes; une convergence de $50 \mathrm{~cm}$ ne perturbe pas les parois d'une galerie de la même façon qu'une convergence de $5 \mathrm{~cm}$. En somme, est-ce la forte convergence qui entraîne la forte fissuration, ou la forte fissuration qui entraîne la forte convergence? Qui a fait I'œuf, qui a fait la poule? II est certainement difficile aujourd'hui de trancher, même si les observations du fluage des calcschistes, notamment autour du revêtement de l'ancienne galerie ferroviaire, peuvent permettre d'espérer une harmonisation des contraintes avec le poids des terres. D'autant plus que les deux explications ne s'opposent pas et peuvent même se superposer. Ne pouvant lever cette ambiguïté, il faudra en tenir compte dans l'interprétation.

Pour tenter de comprendre ce qui s'est produit au sein du massif dans les zones de forte convergence, il est souhaitable évidemment de disposer d'un peu plus d'informations que les simples variations diamétrales. On va donc examiner les sections où des mesures en profondeur ont été faites. Bien entendu elles ont été implantées en fonction des risques pressentis et pas obligatoirement en fonction des convergences rencontrées. On a réussi cependant, en Italie, à obtenir une section vraiment intéressante avec une convergence moyenne de l'ordre de $30 \mathrm{~cm}$ (fig. 3 ). Il est remarquable que du côté France des résultats analogues ont été obtenus; la figure 4 reproduit des mesures publiées par E. Tincelin (1978) et elles sont tout à fait 

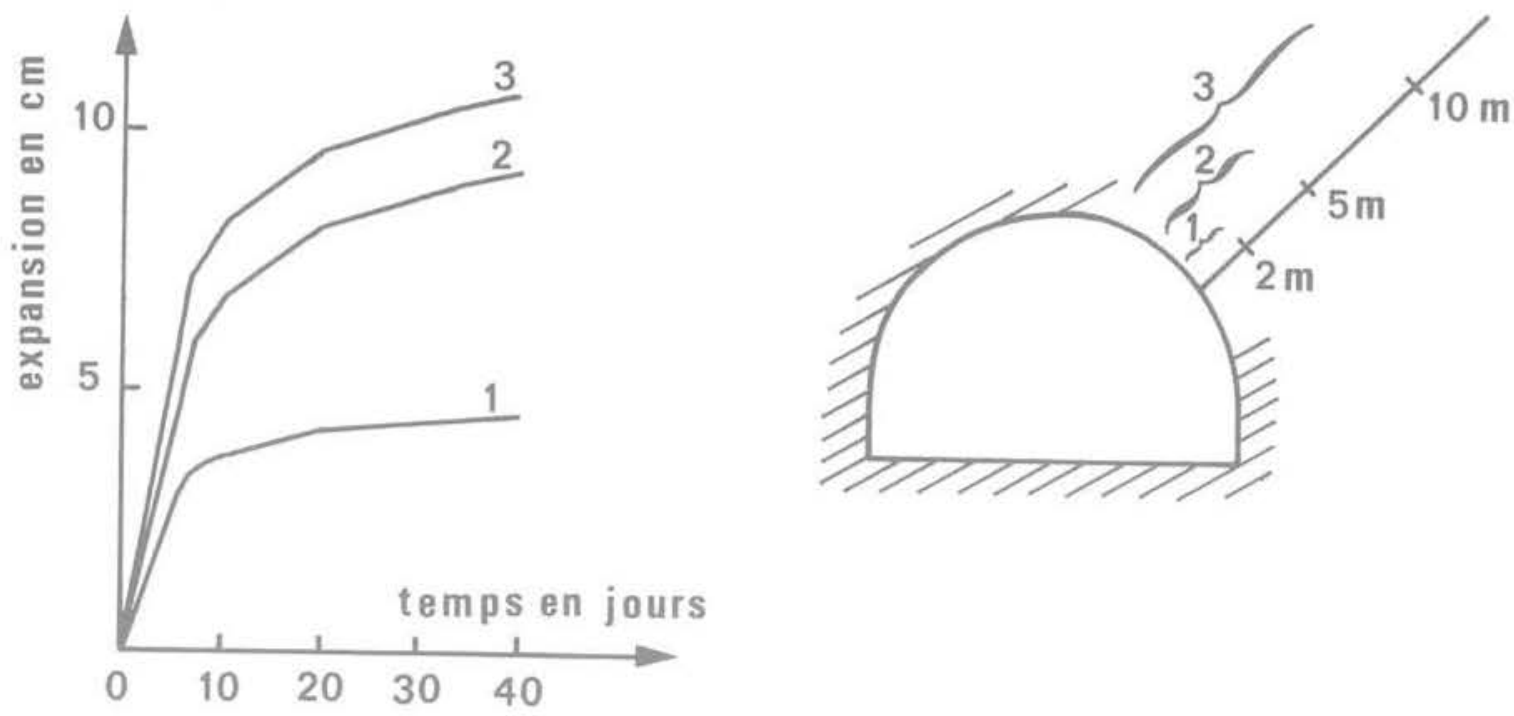

Fig. 4 Mesures d'expansion au tunnel du Frejus (d'après E. Tincelin 1978).

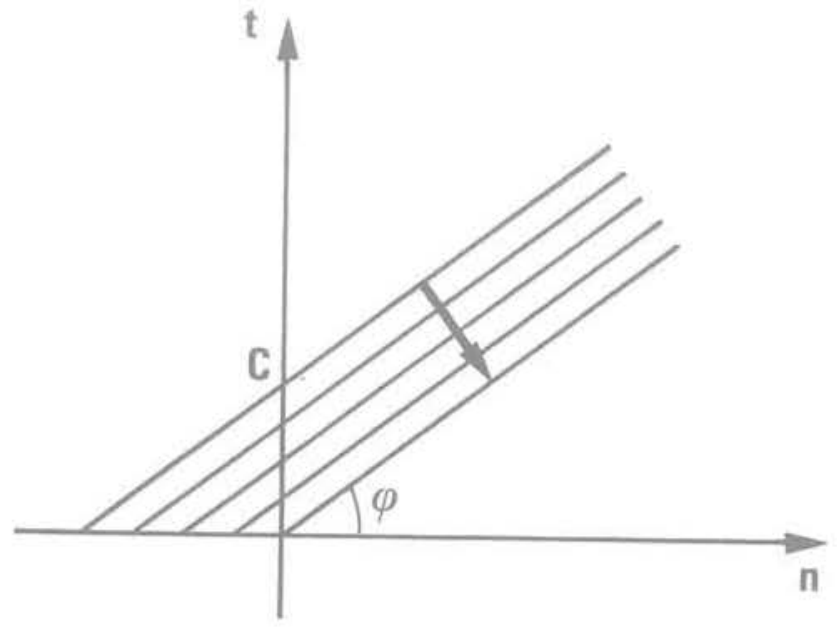

Critère de rupture

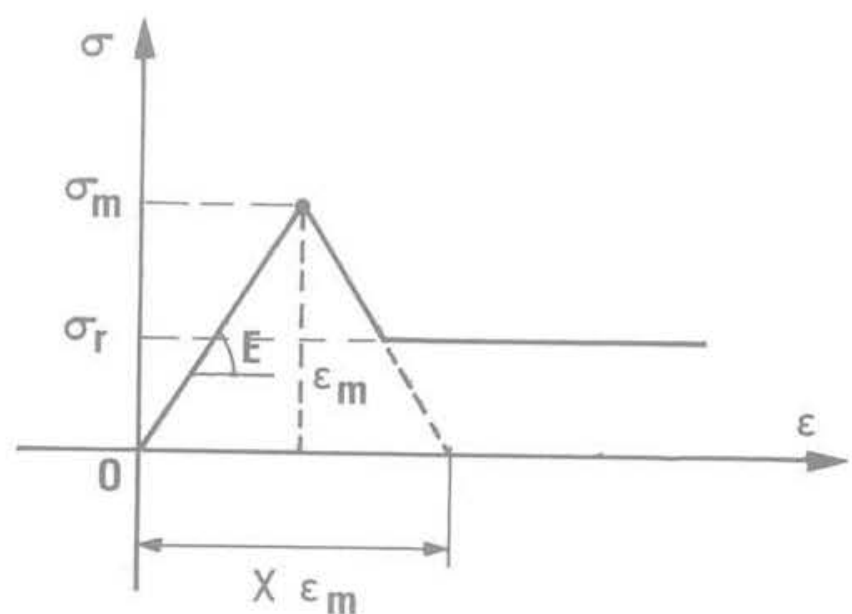

Courbe contrainte-déplacement

Fig. 5 Schématisation du comportement radoucissant.

comparables à celles qui sont présentées ici. La courbe moyenne en pointillé de la figure 3 est la courbe de référence à partir de laquelle l'interprétation va maintenant être faite.

On constate une certaine discondance entre la courbe théorique et ce qui est mesuré, mais il ne faut pas s'en formaliser trop, d'une part parce que le comportement théorique contient une part de schématisation probablement excessive, d'autre part parce qu'il s'agit de mesures in situ toujours délicates à faire avec des possibilités d'erreurs, un scellement peut glisser ou une tige se coincer partiellement au cours de la déformation du rocher.
On sait que le comportement des calcschistes présente un radoucissement; MM. Berest et Nguyen Minh Duc ayant traité assez complètement l'équilibre d'un souterrain dans un tel milieu, il était particulièrement intéressant de comparer la théorie et les mesures. Le comportement théorique radoucissant du milieu est schématisé sur la figure 5 et il est défini par les paramètres $\mathrm{E}, \nu, \varphi, \mathrm{C}$ et $\chi$, paramètre fonction du radoucissement (Berest et Nguyen Minh Duc, 1979). Des vérifications numériques ont montré que certains paramètres ont peu d'importance (la dilatance, le coefficient de Poisson); certains ont une signification physique douteuse $(\chi)$ et on a pu même se demander si 


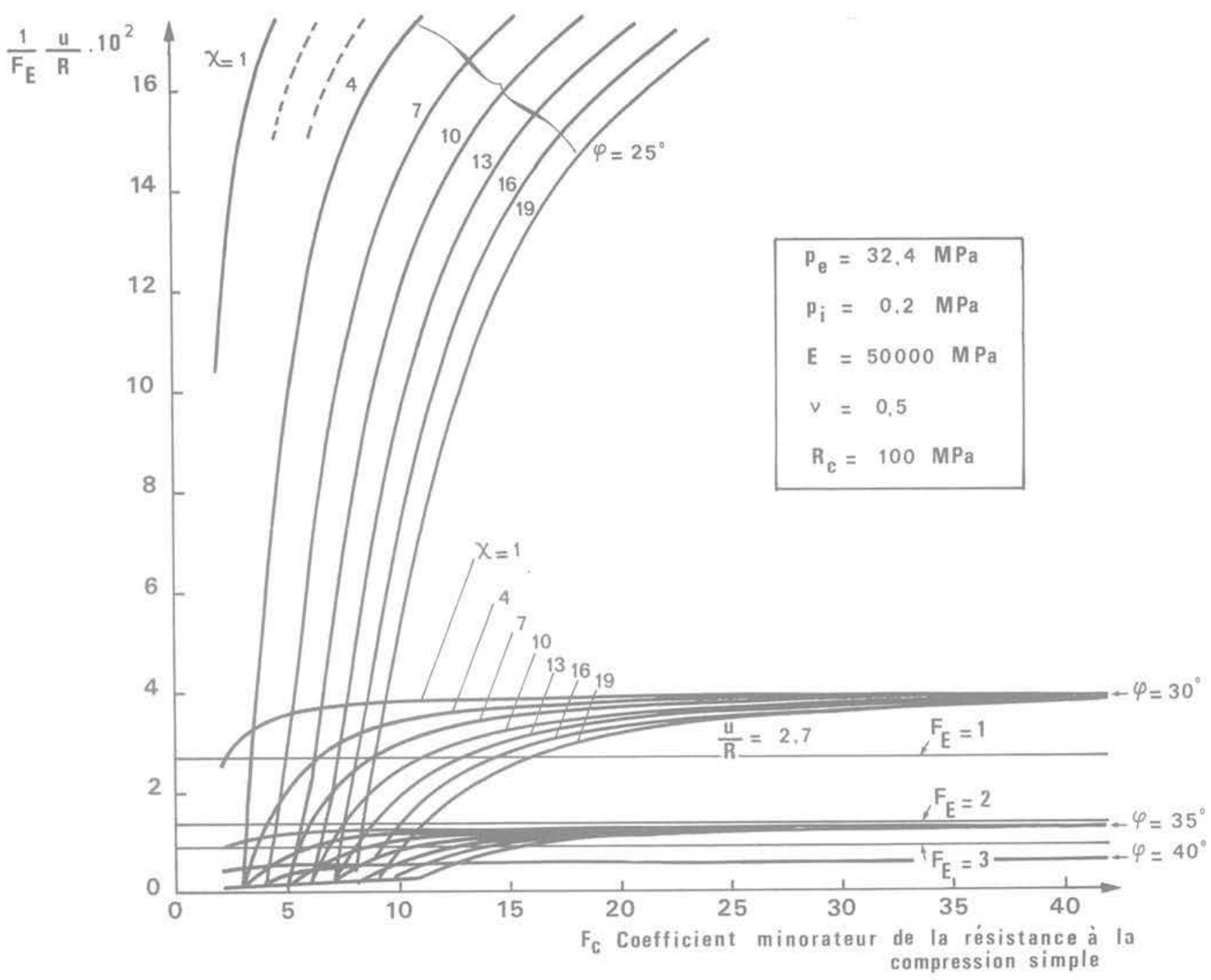

Fig. 6 Schéma élastoplastique radoucissant (tunnel du Fréjus).

la notion de radoucissement se maintenait lorsque le nombre des surfaces de glissement, le nombre des fissures actives, devenait très grand ( $P$. Habib, 1979), et si dans un tel cas on n'était pas ramené au problème élastoplastique classique tel qu'il a été traité, par exemple par J. Salençon (1969). De toutes façon, la comparaison des deux théories, élastoplastique parfaite et élastoplastique avec radoucissement, paraît particulièrement intéressante.

On peut admettre les conditions aux limites suivantes : le massif est indéfiní; le tunnel a la forme d'un trou circulaire de $11 \mathrm{~m}$ de diamètre, le soutènement par boulonnage est équivalent à une pression interne de $0,2 \mathrm{MPa}$; le poids des terres, pour une hauteur de recouvrement de $1200 \mathrm{~m}$ avec une densité de 2,7 correspond à une pression hydrostatique de $32,4 \mathrm{MPa}$. Mais ce n'est pas encore suffisant et il faut faire encore quelques hypothèses pour la valeur de la contrainte longitudinale qui peut, en particulier, créer des conditions d'écoulement en régime de face ou en régime d'arête, ce qui complique le problème. En définitive et en première approximation, on peut accepter une simplification raisonnable en supposant le milieu incompressible $(v=0,5)$, ce qui permet d'éviter le régime d'arête. Mais soulignons que
I'analyse complète reste possible, même en tenant compte de la compressibilité élastique et de la dilatance plastique; l'intérêt de cette simplification pour le calcul est explicité en annexe.

Compte tenu du nombre de variables, la comparaison n'est pas facile, même avec les formules explicites de Berest et Nguyen Minh Duc, et il a fallu recourir à des abaques obtenues par ordinateur pour présenter les résultats en tenant compte de l'incertitude sur le module d'élasticité, sur la résistance à la compression simple, l'angle de frottement interne, la pente du radoucissement, et les contraintes in situ. La figure 6 indique les résultats théoriques pour des angles de frottement interne variant de 25 à $40^{\circ}$ et pour les paramètres d'écrouissage $\chi$ variant de 1 (rupture brutale) à 19 (valeur importante qui rapproche du schéma élastoplastique parfait). En abscisses on a porté un coefficient $F_{c}$ minorateur de la résistance à la compression simple mesurée au laboratoire, prise égale à $100 \mathrm{MPa}$. $\mathrm{F}_{\mathrm{c}}$ représente l'effet d'échelle sur la résistance. En ordonnée, on a porté l'expression :

$$
100 \cdot \frac{1}{F_{E}} \cdot \frac{u}{R}
$$




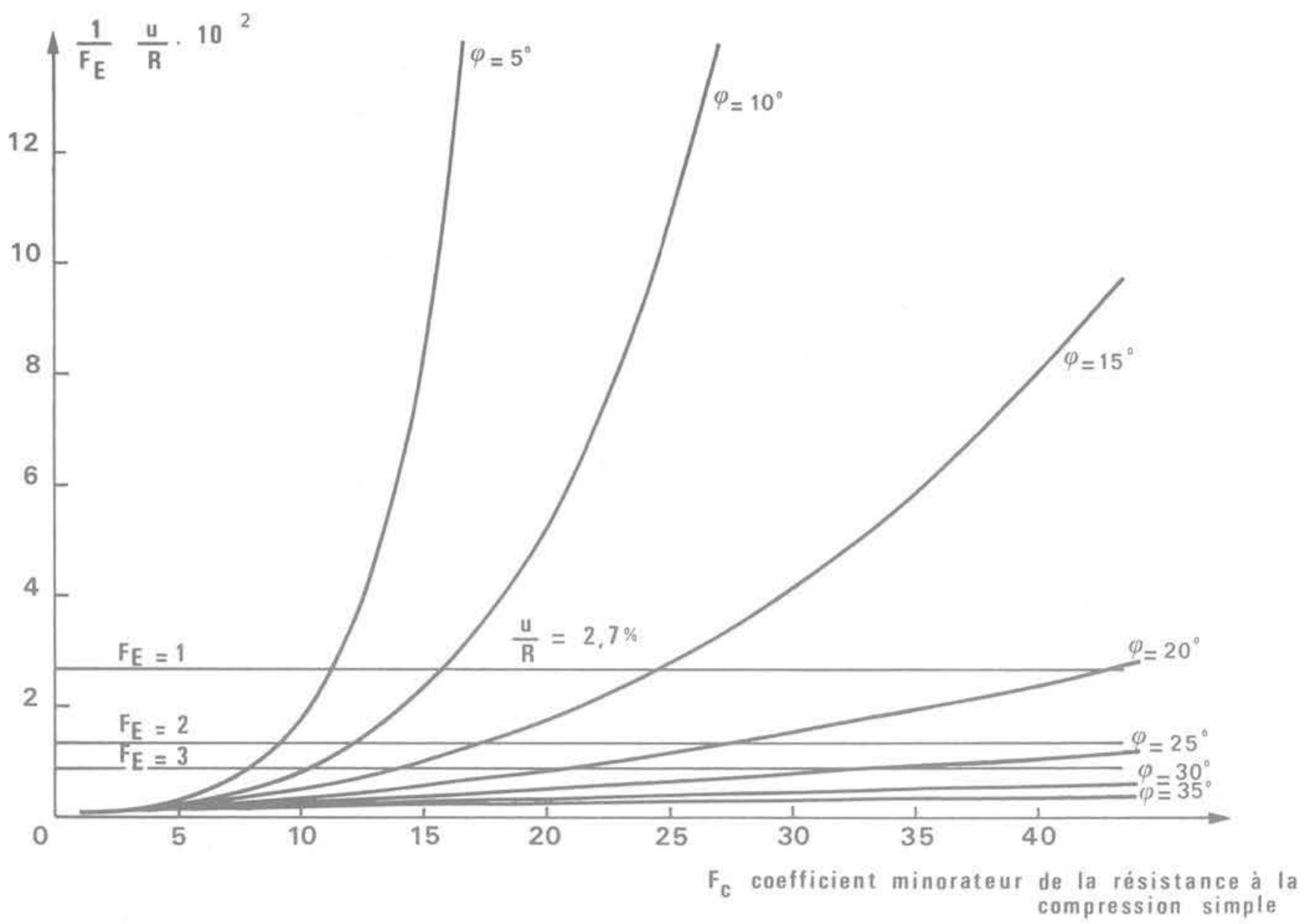

Fig. 7 Schéma élastoplastique parfait (tunnel du Fréjus).

où $\mathrm{u}$ est le déplacement de la paroi, $\mathrm{R}$ le rayon du tunnel et $F_{E}$ le coefficient minorateur représentant l'effet d'échelle sur le module d'élasticité. La déformation de référence $\frac{\mathrm{u}}{\mathrm{R}}$ correspondant à la courbe moyenne de la figure 3 , qui est théoriquement une hyperbole équilatère, est de $2,7 \%$. On voit qu'avec une minoration raisonnable $F_{E}=2$, on obtient pour $\chi=4$ une minoration acceptable de 3 à 4 pour $F_{c}$, lorsque $\varphi$ est compris entre $25^{\circ}$ et $35^{\circ}$.

La figure 7 indique les résultats théoriques pour le schéma élastoplastique parfait dans les mêmes axes. Avec un coefficient minorateur $F_{E}=3$ on ne peut trouver de solution lorsque $\varphi>25^{\circ}$ qu'avec des minorations de résistance $F_{c}>30$, ce qui est tout à fait inacceptable. L'interprétation des observations réelles ne paraît ainsi possible qu'avec le schéma radoucissant, mais pas avec le schéma élastoplastique classique.

On a vu cependant qu'une incertitude existait vis-à-vis des contraintes naturelles qui règnent sur le tracé du souterrain. La figure 8 indique les résultats théoriques obtenus avec le schéma radoucissant en portant en abscisses un coefficient majorateur $F_{h}$ du recouvrement; l'axe des Ordonnées est le même que celui des figures 6 et 7 ; le calcul initial est fait en supposant un recouvrement de $1200 \mathrm{~m}$. Avec un minorateur raison- nable du module d'élasticité $F_{E}=2$ et pour $\chi=4$, les résultats obtenus sont expliqués par une majoration de 1,5 à 2 de la pression initiale dans le massif lorsque $\varphi$ varie de $30^{\circ}$ à $40^{\circ}$. Là encore une interprétation acceptable peut être trouvée dans le cadre théorique du radoucissement. La figure 9 indique la même analyse avec le schéma élastoplastique parfait, avec les mêmes axes que ceux de la figure 8 . Un coefficient majorateur du recouvrement de 5 à 6 est nécessaire pour $F_{E}=2$ lorsque $\varphi$ varie de 30 à $40^{\circ}$, et une telle mémoire des contraintes tectoniques paraît véritablement invraisemblable pour les calcschistes du Mt Fréjus, ce qui montre à nouveau que le schéma élastoplastique classique est insuffisant pour interpréter les observations faites dans le tunnel.

\section{b) Tunnel du Gran Sasso}

Tout ce qui vient d'être dit pour le tunnel du Fréjus est valable pour le tunnel du Gran Sasso car les deux ouvrages sont très semblables, tant au point de vue des dimensions intérieures que de l'épaisseur du recouvrement. En particulier, étant donné l'imprécision de l'évaluation du module d'élasticité, l'hypothèse de l'isotropie du massif est valable en première approximation. De même l'homogénéité des marnes est une supposition acceptable dans les mêmes conditions. 


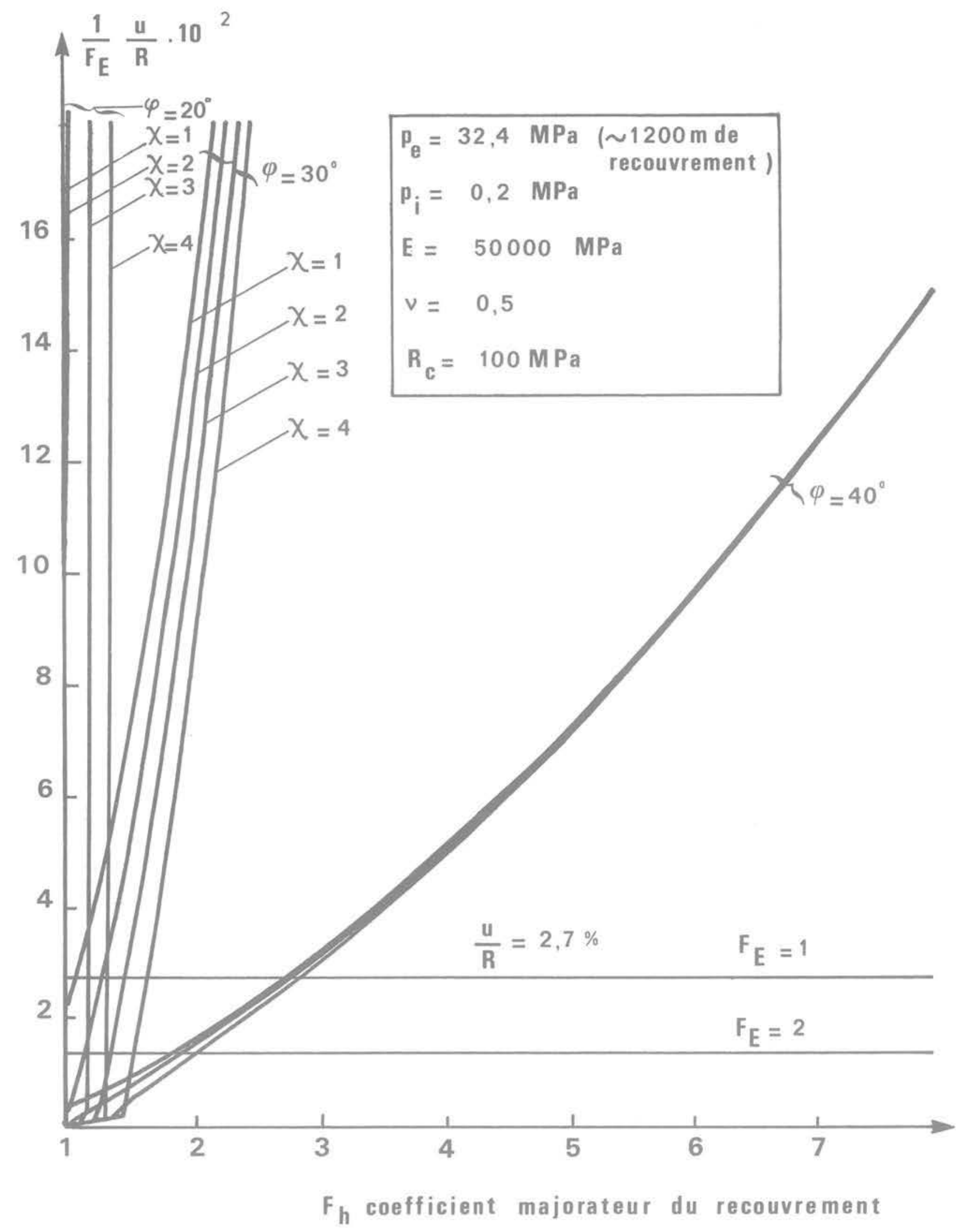

Fig. 8 Influence des contraintes résiduelles; schéma élastoplastique radoucissant (tunnel du Fréjus). 


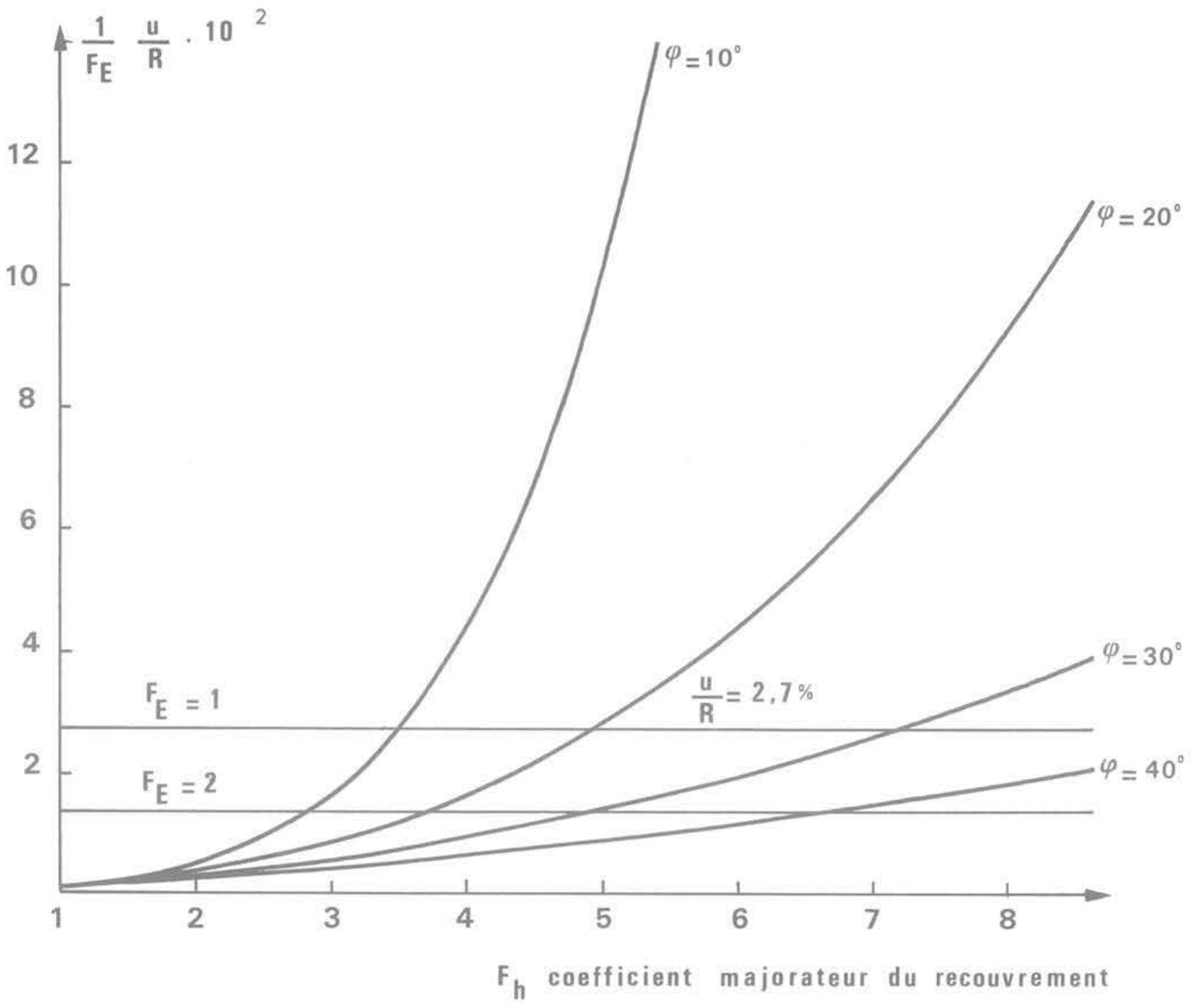

Fig. 9 Influence des contraintes résiduelles; schéma élastoplastique parfait (tunnel du Fréjus).

L'analyse de la comparaison entre les observations dans le tunnel et les résultats théoriques peut donc être faite très rapidement.

La figure 10 indique les déformations en profondeur autour du tunnel sous un recouvrement de $900 \mathrm{~m}$ de marne de densité 2,5 , équivalent à une pression hydrostatique de $22,5 \mathrm{MPa}$. Les mesures ont été effectuées par Baldovin, Lunardi et Al (1977).

La courbe moyenne des déplacements en profondeur autour du tunnel, en pointillé sur la figure 10, est une hyperbole; le point sur la paroi du tunnel correspond à une déformation de référence $\frac{\mathrm{u}}{\mathrm{R}}=2,4 \%$.

Les mêmes auteurs ont effectué des essais in situ pour la détermination des propriétés mécaniques du massif. L'estimation de l'élasticité et de la résistance de la marne a été faite à partir d'essais sur des blocs de volume important, de sorte les caractéristiques mécaniques suivantes peuvent être acceptées sans effet d'échelle (ou tout au moins avec des effets d'échelle très limités, c'est-à-dire $F_{E}$ et $F_{c}$ voisins ou légèrement supérieurs à l'unité) :

$$
\begin{aligned}
\mathrm{R}_{\mathrm{c}} & =4,5 \mathrm{MPa} \\
\mathrm{E} & =3000 \mathrm{MPa} \\
\varphi & =30^{\circ} .
\end{aligned}
$$

Avec ces valeurs et, comme pour le tunnel du Fréjus, en simplifiant avec les hypothèses de l'incompressibilité élastique $(v=0,5)$ et de l'invariance du volume en régime plastique, avec des valeurs raisonnables pour la pente du radoucissement on peut tenter d'interpréter les observations avec les deux théories, plasticité parfaite ou radoucissement. 

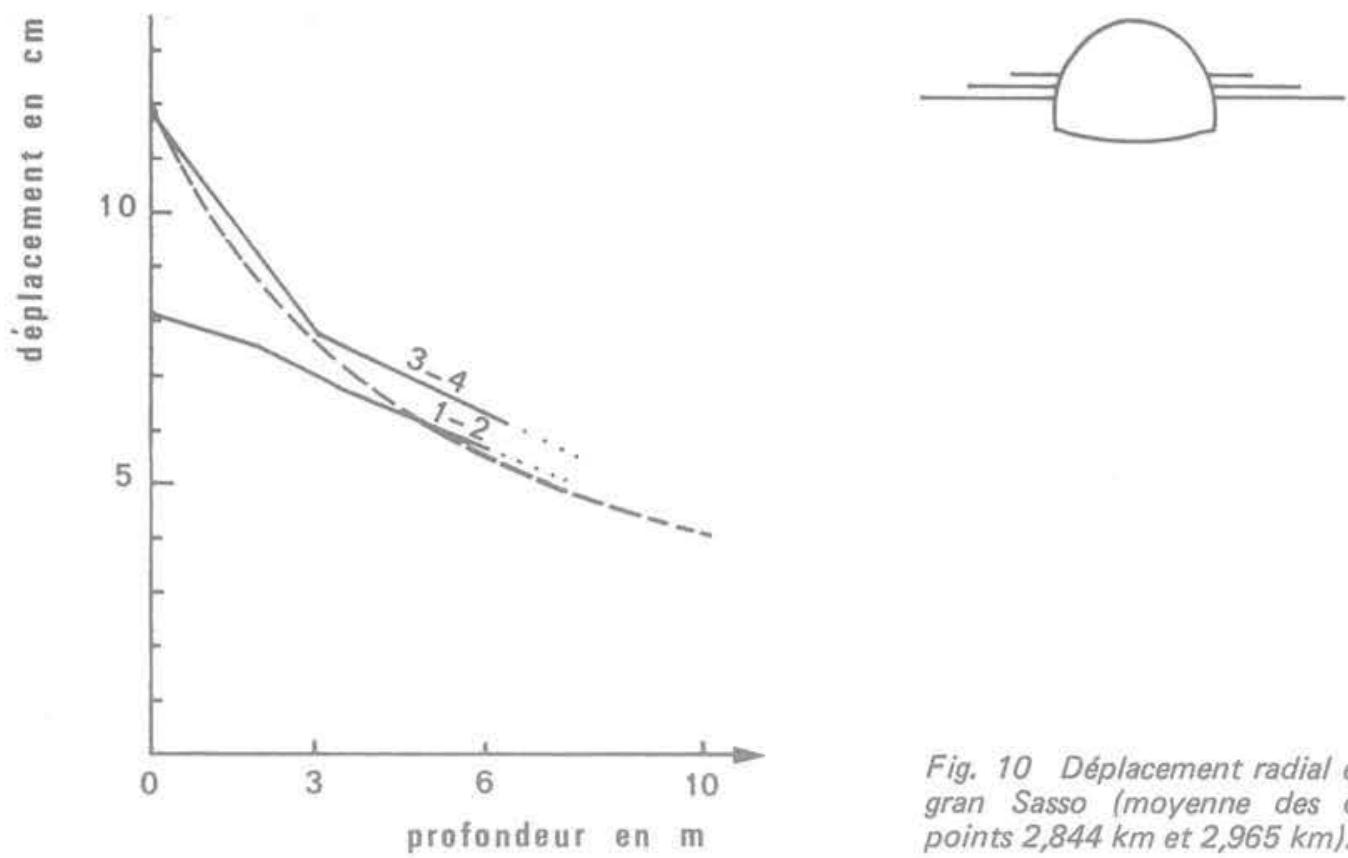

Fig. 10 Déplacement radial en profondeur au tunnel du gran Sasso (moyenne des déplacements mesurés aux points $2,844 \mathrm{~km}$ et $2,965 \mathrm{~km})$.

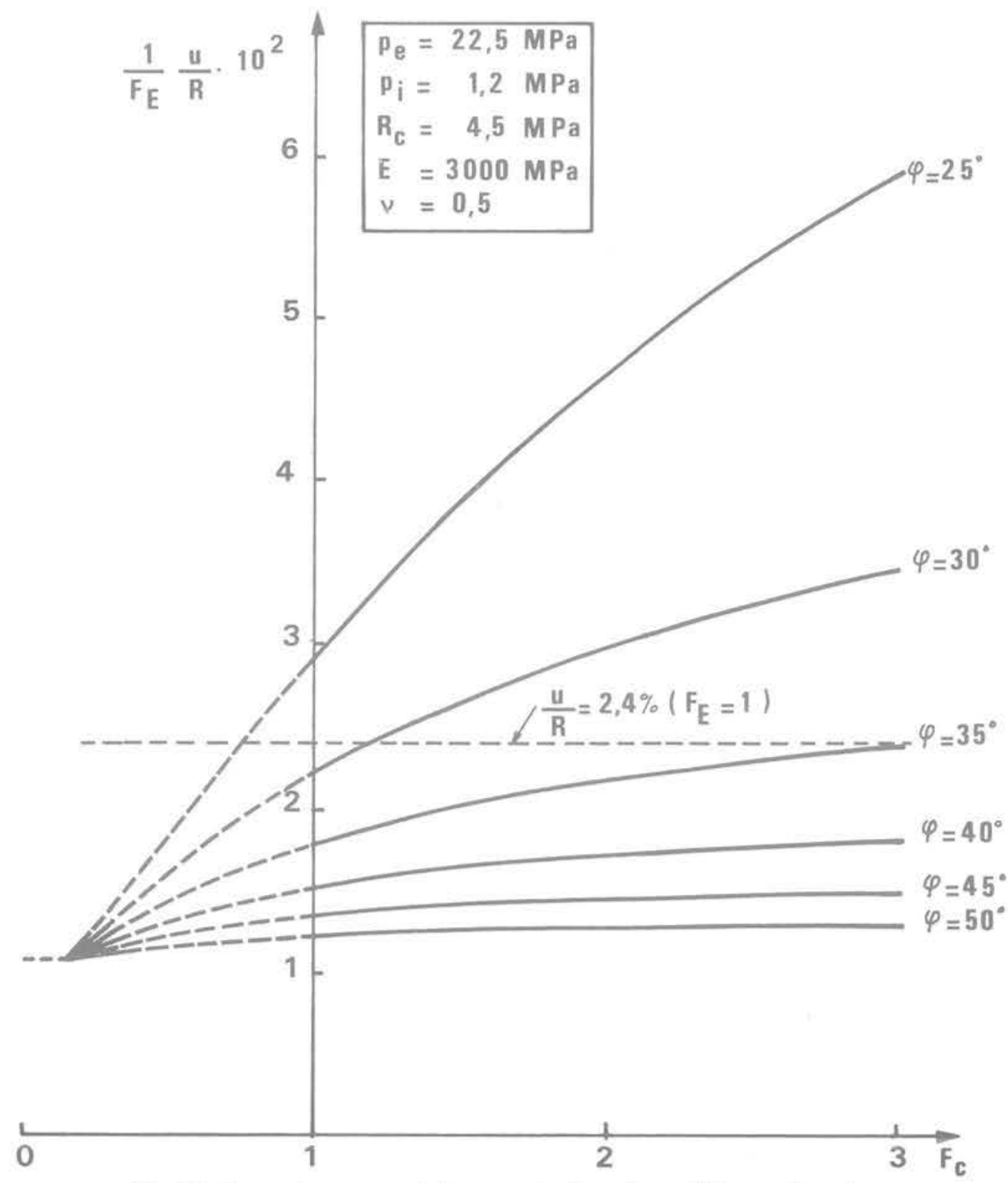

Fig. 11 Comportement parfaitement plastique (tunnel du gran Sasso). 


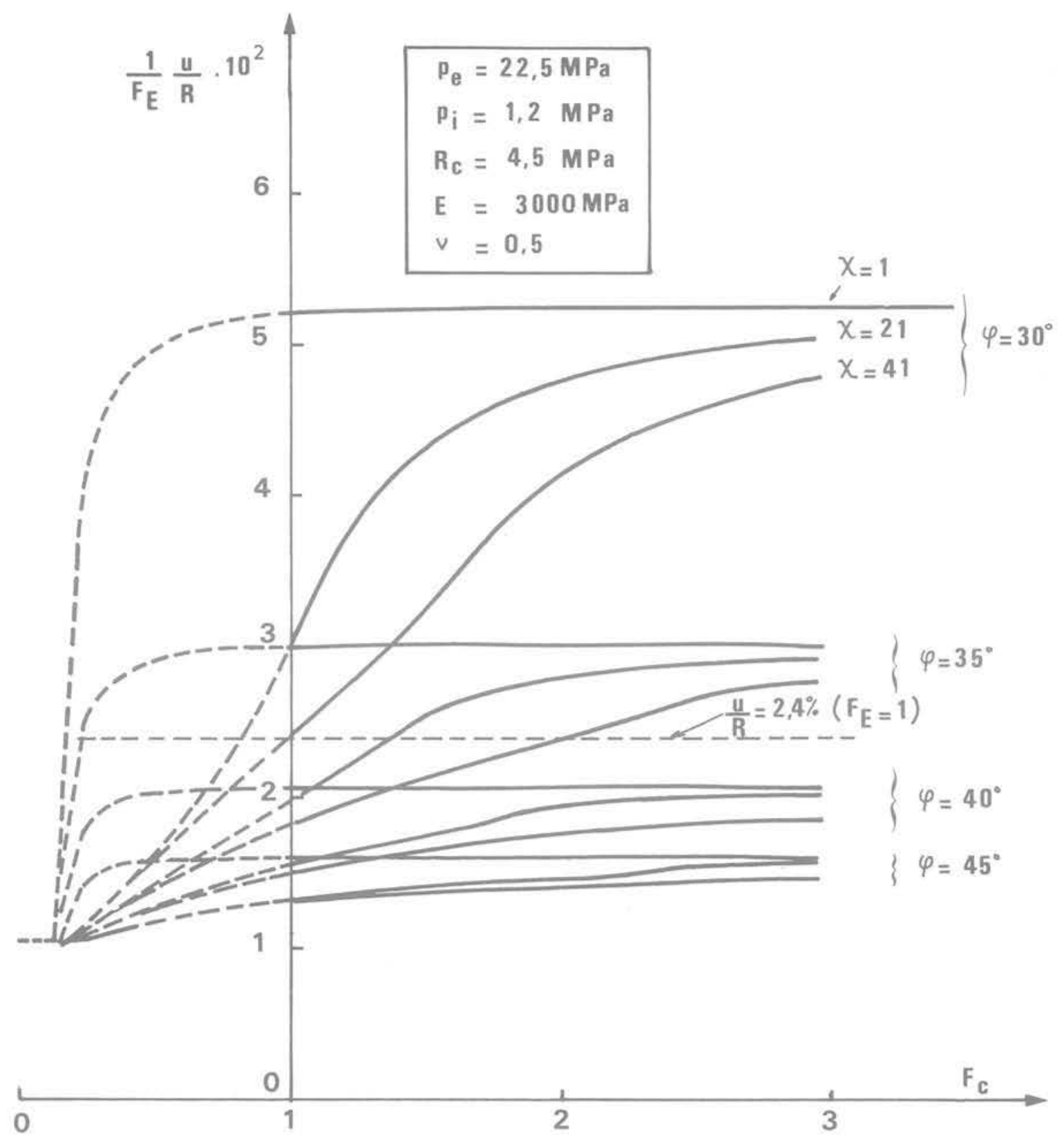

Fig. 12 Comportement radoucissant (tunnel du gran Sasso).

La figure 11 indique avec les mêmes axes que ceux de la figure 7 les résultats obtenus en plasticité parfaite. Les observations dans le tunnel avec $F_{E}$ et $F_{c}$ voisins de 1 sont en accord avec l'abaque. Par contre la figure 12 qui représente l'interprétation élastoplastique avec radoucissement, avec la même présentation que la figure 6 , ne permet pas de trouver un accord satisfaisant avec les mesures en place. Sur cet abaque on a indiqué les résultats obtenus pour des valeurs de $\chi$ très grandes qui rapprochent le schéma radoucissant du schéma plastique parfait. On voit qu'à la limite les résultats sont confondus.

\section{Conclusion}

L'interprétation des mesures de déplacements faites dans les tunnels du Gran Sasso et du Fréjus montre clairement que deux théories sont nécessaires. La théorie élastoplastique avec radoucissement permet d'expliquer ce qui s'est passé au tunnel du Fréjus, alors que la théorie parfaitement plastique ne le permet pas. C'est l'inverse au tunnel du Gran Sasso. Cette constatation étant faite, elle paraît assez naturelle a posteriori, les calcschistes du Fréjus ayant au laboratoire un comportement nettement fragile alors que le comportement des marnes du Gran Sasso est certainement beaucoup plus plastique.

Dans l'un et l'autre cas les calculs faits ont négligé des phénomènes importants : l'action de la pesanteur dans le voisinage immédiat du tunnel, l'anisotropie du massif, son hétérogénéité, etc. Les résultats obtenus sont cependant suffisamment nets pour démontrer le rôle capital joué par le radoucissement dans certains cas et la nécessité de connaître le comportement mécanique complet pour la prévision de déformation et des soutènements des galeries souterraines. 


\section{Annexe}

\section{Analyse de la convergence des tunnels par des modèles élastoplastiques}

Sur le modèle géométrique du tunnel on définit les données de base suivantes:

h - hauteur du recouvrement au-dessus du tunnel (équivalente à une pression $\mathrm{p}_{\mathrm{e}}$ uniforme à l'infini);

$p_{i}$ - pression à la paroi de la cavité équivalente à la réaction du soutènement;

R - rayon du cylindre qui s'ajuste au plus près de la forme du tunnel.

Les données mécaniques classiques, susceptibles d'être obtenues expérimentalement sont les suivantes :

$R_{c}$ - résistance à la compression simple;

E - module de Young:

$\varphi$ - angle de frottement interne.

En utilisant la théorie de l'élastoplasticité parfaite, d'une part, celle de l'élastoplasticité avec radoucissement, d'autre part, théories rappelées dans le texte, on cherche à déterminer des paramètres $h^{\prime}, R_{c}^{\prime}, E^{\prime}$ ou $\varphi^{\prime}$ qui donnent un accord satisfaisant avec les convergences mesurées. L'analyse porte sur la vraisemblance des rapports entre ces grandeurs et les grandeurs mesurées. On obtient ainsi les coefficients majorateurs correctifs

$$
\mathrm{F}_{\mathrm{h}}=\frac{\mathrm{h}^{\prime}}{\mathrm{h}} ; \quad \mathrm{F}_{\mathrm{c}}=\frac{\mathrm{R}_{c}}{\mathrm{R}_{\mathrm{c}}^{\prime}} ; \quad \mathrm{F}_{\mathrm{E}}=\frac{\mathrm{E}}{\mathrm{E}^{\prime}} ; \quad \mathrm{F}_{\varphi}=\frac{\varphi}{\varphi^{\prime}}
$$

que I'on comparera pour les deux modèles.

\section{Hypothèse d'incompressibilité du matériau dans les modèles}

Cette hypothèse est une première approximation acceptable dont la mesure où le calcul montre que des variations de volume raisonnables au cours de la déformation ne modifient pas considérablement la valeur des convergences. Par contre cette hypothèse présente un double intérêt :

- le régime d'arête n'apparaît pas $(v=0,5)$ dans l'écoulement plastique;

- le déplacement radial $u(y)$, en fonction du rayon de la zone plastique y, a la même expression pour les deux théories:

$$
E \frac{u}{r}=\frac{3}{2} \frac{p_{e}\left(K_{p}-1\right)+R_{c}}{K_{p}+1} \frac{y^{2}}{r^{2}}
$$$$
\text { ou่ : } \quad K_{p}=\operatorname{tg}^{2}\left(\frac{\pi}{4}+\frac{\varphi}{2}\right) \text {. }
$$

En fonction de $r, E \frac{u}{r}$ est une courbe en $\frac{A}{r^{2}}$; en ajustant le déplacement $u$ en fonction de $r$ aux courbes tracées à partir des mesures in situ, on obtient le paramètre A qui définit une déformation caractéristique à la paroi; y étant exprimé en fonction des paramètres des modèles, on obtient l'ensemble des coefficients correctifs possibles $F$, qu'on a calculés de façon à les présenter sous formes d'abaques.

On remarquera que le deuxième membre de (1) ne dépend pas du module d'Young, ce qui simplifie la présentation pour $F_{E}$.

\section{Calcul du rayon plastique}

Pour le modèle parfaitement plastique, on obtient la forme explicite :

$$
y=R\left[\frac{2}{K_{p}+1} \cdot \frac{p_{e}\left(K_{p}-1\right)+R_{c}}{p_{i}\left(K_{p}-1\right)+R_{c}}\right]^{\frac{1}{p-1}}
$$

Pour le modèle élastoplastique radoucissant la loi de comportement choisie conduit à distinguer le cas où la zone plastique purement radoucissante (zone 1) apparaît seule, du cas où apparaît aussi une zone 2 parfaitement plastique à cohésion nulle.

$\alpha_{1}$ et $\alpha_{2}$ (définis par Nguyen M. D. et Berest, 1979) ont ici des valeurs simples:

$$
\alpha_{1}=-2 \quad \alpha_{2}=\frac{x}{x-\frac{3}{4}}\left(K_{p}-1\right) .
$$

La déformation plastique équivalente à la paroi vaut alors, en phase radoucissante

$$
\begin{aligned}
\lambda(R, y)=\frac{3}{2} \frac{p_{e}\left(K_{p}-1\right)+}{R_{c}} & \cdot \frac{1}{x\left(K_{p}+1\right)-\frac{3}{2}} \cdot\left\{\left(\frac{y}{R}\right)^{2}-\left(\frac{y}{R}\right)^{-x_{2}}\right\}
\end{aligned}
$$

On a donc :

a) $\lambda(R, y)<1$ : la zone 1 radoucissante existe seule

(3)

$$
\begin{aligned}
\frac{x\left(K_{p}+1\right)-\frac{3}{2}}{\frac{3}{4}\left(K_{p}-1\right)\left(\frac{y}{R}\right)^{2}+\left(K_{p}+1\right)\left(x-\frac{3}{4}\right)\left(\frac{y}{R}\right)^{-\alpha_{2}}} \\
=\frac{2}{K_{p}+1} \cdot \frac{p_{e}\left(K_{p}-1\right)+R_{c}}{p_{1}\left(K_{p}-1\right)+R_{c}} .
\end{aligned}
$$

On retrouve sur cette formule, par passage à la limite, le cas de la plasticité parfaite (formule 2); en effet : $x \longrightarrow \infty, \alpha_{2} \longrightarrow \mathrm{K}_{\mathrm{p}}-1$.

b) $\lambda(R, y)>1$ : les deux zones 1 et 2 sont apparues

Soit $y_{1}$ la solution de $\lambda(y)=1$.

Le rayon de la zone plastique a alors pour expression :

$$
\begin{aligned}
& y=y_{1}\left[\frac{2}{K_{p}+1} \frac{P_{e}\left(K_{p}-1\right)+R_{c}}{P_{1}\left(K_{p}-1\right)+R_{c}}\left(\frac{y_{i}}{R}\right)^{2}\right. \\
& \left.-\frac{R_{c}}{p_{i}\left(K_{p}-1\right)+R_{c}}\left(\frac{4}{3} x-1\right)\right]^{K_{p-1}}
\end{aligned}
$$

\section{Références bibliographiques}

BALDOVIN G., LUNARDI P., CATALANO P. G., MOTTA E. (1977), A deep tunnel in highly tectonized marl. The Geotechnics of Structurally Complex Formations, Capri, 1977.

BEREST P., NGUYEN MINH D., PANET M. (1978), Contribution à l'étude de la stabilité d'une cavité souterraine dans un milieu avec radoucissement. Revue Française de Géotechnique, $n^{\circ} 4$, juin 1978 , p. 63. 
HABIB P. (1979), Équilibre des souterrains dans les matériaux radoucissants. AFTES: Stabilité des Tunnels, Paris, 26 octobre 1978. Tunnels et Ouvrages Souterrains, $\mathrm{n}^{\circ} 32$, mars-avril 1979, p. 126.

NGUYEN MINH D., BEREST P. (1977), Contraction d'une sphère creuse élastoplastique avec radoucissement. Journal de Mécanique Appliquée, $n^{\circ} 1,1977$, p. 85.

NGUYEN MINH D., BEREST P. (1979), Étude de la stabilité des cavités souterraines avec un modèle de comportement élastoplastique radoucissant. $4^{\circ}$ Congrès de la Société Internationale de Mécanique des Roches, tome I, p. 249, Montreux, septembre 1979.
SALENÇON J. (1969), Contraction quasistatique d'une cavité à symétrie sphérique ou cylindrique dans un milieu élastoplastique. Annales des Ponts et Chaussées, vol. IV, 1969, p. 231.

STRAGIOTTI et Al. (1979), Essais et auscultations dans la conception et l'exécution de quatre tunnels dans les schistes lustrés de la vallée de Suse (Italie). $4^{e}$ Congrès de la Société Internationale de Mécanique des Roches, tome II, p. 663, Montreux, septembre 1979.

TINCELIN E., SINOU P. (1978), Mode d'action et règles du boulonnage. "Le Boulonnage", Document SIM-B5, Revue de I'Industrie Minérale, octobre 1978, p. 129. 
